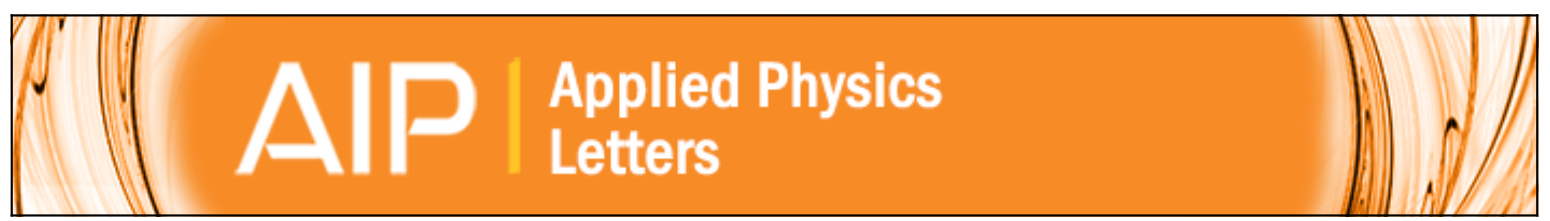

\title{
Electronic structure of single-walled carbon nanotubes on ultrathin insulating films
}

Hyung-Joon Shin, Sylvain Clair, Yousoo Kim, and Maki Kawai

Citation: Applied Physics Letters 93, 233104 (2008); doi: 10.1063/1.3046114

View online: http://dx.doi.org/10.1063/1.3046114

View Table of Contents: http://scitation.aip.org/content/aip/journal/apl/93/23?ver=pdfcov

Published by the AIP Publishing

\section{Articles you may be interested in}

Surface electronic structure of nitrogen-doped semiconducting single-walled carbon nanotube networks J. Appl. Phys. 114, 153516 (2013); 10.1063/1.4826206

Electronic structure and field emission properties of in situ potassium-doped single-walled carbon nanotubes Appl. Phys. Lett. 90, 023108 (2007); 10.1063/1.2431463

Time-related conversion of the carbon nanotube field effect transistor Appl. Phys. Lett. 89, 233507 (2006); 10.1063/1.2402218

Electrochemical gating of individual single-wall carbon nanotubes observed by electron transport measurements and resonant Raman spectroscopy Appl. Phys. Lett. 84, 2052 (2004); 10.1063/1.1666997

Electronic structure of kinked multiwalled carbon nanotubes Appl. Phys. Lett. 76, 3594 (2000); 10.1063/1.126717

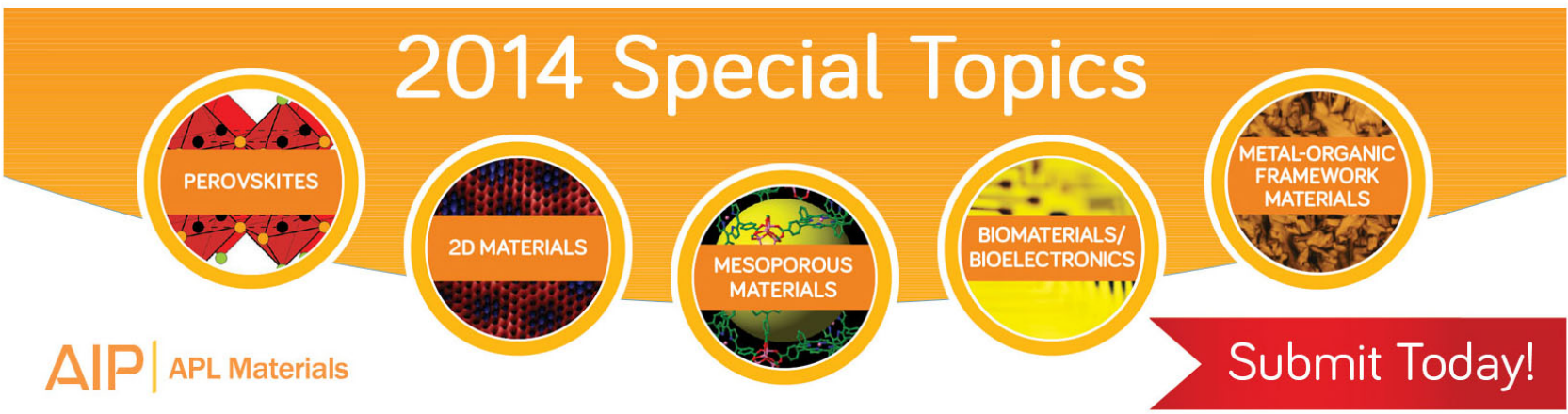




\title{
Electronic structure of single-walled carbon nanotubes on ultrathin insulating films
}

\author{
Hyung-Joon Shin, ${ }^{1,2, a)}$ Sylvain Clair, ${ }^{1,3}$ Yousoo Kim, ${ }^{1, b)}$ and Maki Kawai ${ }^{1,2,3, b)}$ \\ ${ }^{1}$ Surface Chemistry Laboratory, RIKEN, Wako, Saitama 351-0198, Japan \\ ${ }^{2}$ Department of Applied Chemistry, The University of Tokyo, 7-3-1 Hongo, Bunkyo-ku, \\ Tokyo 113-8656, Japan \\ ${ }^{3}$ Department of Advanced Materials Science, The University of Tokyo, 5-1-5 Kashiwanoha, Kashiwa, \\ Chiba 277-8561, Japan
}

(Received 1 October 2008; accepted 20 November 2008; published online 9 December 2008)

\begin{abstract}
The electronic structures of single-walled carbon nanotubes on $\operatorname{Ag}(100)$ and on ultrathin insulating $\mathrm{NaCl}(100) / \mathrm{Ag}(100)$ were studied using low-temperature scanning tunneling microscopy. The Fermi level of the nanotubes was shifted toward the conduction band on $\operatorname{Ag}(100)$, while it was shifted toward the valence band on $\mathrm{NaCl}$ films. We explain this opposite behavior by different basic mechanisms accounting for the Fermi level shifts. On the metal surface, the work function difference between the tube and the substrate determines the direction of the Fermi level shift. In the case of carbon nanotubes on insulating films, the electric field resulting from the dipole moment formed at the interface between the insulating film and the metal plays a decisive role in determining the Fermi level. (0) 2008 American Institute of Physics. [DOI: 10.1063/1.3046114]
\end{abstract}

Single-walled carbon nanotubes (SWCNTs) are undoubtedly one of the most promising materials for future development of nanoscale electronic devices. ${ }^{1,2}$ To make such devices possible, it is necessary to acquire a detailed understanding on the behavior of a SWCNT in contact with various materials. Low-temperature scanning tunneling microscopy (LT-STM) and scanning tunneling spectroscopy (STS) are especially well suited to studying local electronic structures with atomic resolution. ${ }^{3-5}$ Most experiments on SWCNT devices have been carried out on insulating layers for practical applications; to date, however, STM studies have been restricted to SWCNTs on metals ${ }^{3-5}$ or semiconductors. ${ }^{6,7}$ In the case of insulating films of only a few atomic layers in thickness, electrons can tunnel through the film while the hybridization that takes place between a molecular adsorbate and a conducting substrate could be substantially reduced for STM study. ${ }^{8-10}$ However, due to the difficulties in preparing SWCNTs on insulating films in the extremely clean conditions required for STM study in ultrahigh vacuum (UHV) environment, the electronic structure of SWCNTs on insulating films has not yet been a focus of study. In this letter, we report on our study of the electronic structure of SWCNTs on $\mathrm{NaCl}(100) / \mathrm{Ag}(100)$ by STM and STS. We were able to prepare SWCNTs by employing a dry contact transfer (DCT) technique, a method for depositing SWCNTs on various kinds of substrates. ${ }^{6,7,11}$ Our results show the importance of the substrate on the electronic structure of SWCNTs, especially the Fermi level $\left(E_{F}\right)$ alignment. With regard to the opposite behavior observed, we discuss on the origin of the $E_{F}$ shift on metal substrate and on insulating film.

The experiments were carried out in an UHV lowtemperature scanning tunneling microscope (Omicron LTSTM) at $4.7 \mathrm{~K}$. The $\mathrm{Ag}(100)$ single crystal was cleaned by

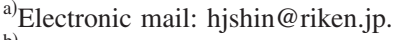

b) Authors to whom correspondence should be addressed. Electronic addresses: ykim@riken.jp and maki@riken.jp.
}

several cycles of sputtering and annealing. After sample cleaning, the $\mathrm{NaCl}$ film was deposited on the substrate by thermal evaporation at $600 \mathrm{~K}$. Finally, HiPCO SWCNTs (Carbon Nanotechnologies, Inc.) were deposited in situ by the DCT method on the $\mathrm{NaCl} / \mathrm{Ag}(100)$ substrate in an UHV chamber. ${ }^{11}$ By this method, we were able to prepare SWCNTs on the $\mathrm{NaCl} / \mathrm{Ag}(100)$ surface without significant contamination of the samples. In our STM results, all the bias voltage refers to the sample voltage with respect to the tip.

Figure 1(a) shows the $\mathrm{NaCl}$ islands on the $\mathrm{Ag}$ substrate. The $\mathrm{NaCl}$ islands grow in the carpet growth mode on $\mathrm{Ag}(100)$. In general, the nucleation of $\mathrm{NaCl}$ begins from the step site and spreads over both terraces. Figures 1(b) and 1(c) show SWCNTs deposited on the $\mathrm{NaCl}$ films. Isolated SWCNTs were easily found elsewhere on $\mathrm{NaCl}$ islands or on silver by means of STM. In some cases, SWCNTs were placed both on the $\mathrm{Ag}$ substrate and on the $\mathrm{NaCl}$ islands as in Fig. 1(c), where the left side of the tube was positioned over the silver and the right side was placed on $\mathrm{NaCl}$ layers of 2 ML in thickness.

In order to investigate the electronic structure of SWCNTs, we measured differential conductance, $d I / d V$, of SWCNTs on $\mathrm{Ag}(100)$ and on $\mathrm{NaCl}(100)$ films, respectively. The $d I / d V$ was obtained with a lock-in detection by applying a modulation of $50 \mathrm{mV}(\mathrm{rms})$ to the tunneling voltage at $797 \mathrm{~Hz}$. The $E_{F}$ of the SWCNTs on $\operatorname{Ag}(100)$ shifts toward the conduction band $(\mathrm{CB})$, that is, the midgap position is located from about -500 to $-100 \mathrm{meV}$ [Figs. 2(a)-2(c)]. In contrast, the position of $E_{F}$ shifts to the valence band (VB) on the $\mathrm{NaCl}$ film, where the midgap of the SWCNT is located at about $+300-+400 \mathrm{meV}$ [Figs. 2(d)-2(f)]. Regardless of the type of the tubes (semiconducting or metallic), the $E_{F}$ shifts in all SWCNTs were similar to the above results (toward the $\mathrm{CB}$ on the silver and toward the $\mathrm{VB}$ on the $\mathrm{NaCl}$ films). The $E_{F}$ shift toward the VB direction has been reported in many STS studies on SWCNTs on the Au(111) surface. ${ }^{3}$ The most important factor determining the position 

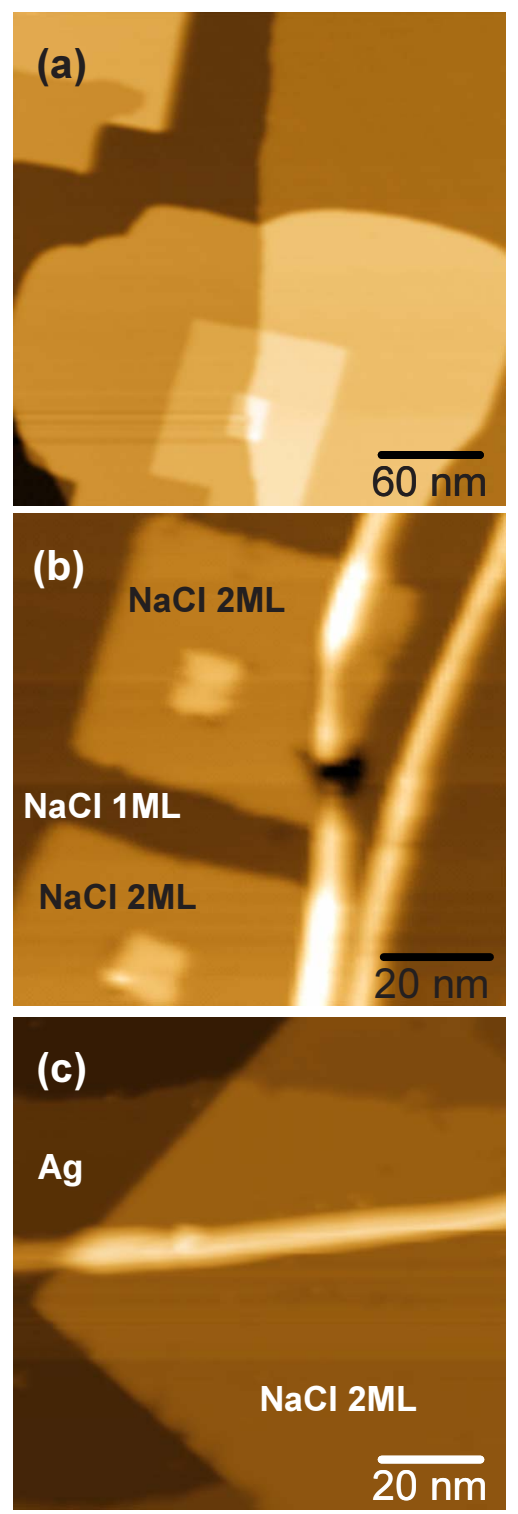

FIG. 1. (Color online) (a) STM image of $\mathrm{NaCl}(100)$ islands on the $\mathrm{Ag}(100)$. [(b) and (c)] STM images of SWCNTs on the $\mathrm{NaCl}$ islands and Ag substrate. The imaging conditions are (a) $V_{S}=1.5 \mathrm{~V}$ and $I_{T}=250 \mathrm{pA}$, (b) $V_{S}=3.5 \mathrm{~V}$ and $I_{T}=250 \mathrm{pA}$, and (c) $V_{S}=1.5 \mathrm{~V}$ and $I_{T}=250 \mathrm{pA}$, respectively.

of $E_{F}$ of a SWCNT on the metal substrate is the difference in work functions (WFs) between the tube and the substrate. ${ }^{12,13}$ This means that the charge transfer between the SWCNT and the metal substrate can be varied according to the WFs of the substrates. Since the WF of $\mathrm{Ag}(100)$ is about $4.4 \mathrm{eV}^{14}$ which is smaller than that of $\mathrm{Au}(111)$ $(5.3 \mathrm{eV}),{ }^{15}$ the charge transfer occurs from the SWCNT to the $\mathrm{Ag}(100)$ and from the $\mathrm{Au}(111)$ to the SWCNT, which is known to have a WF around $4.8 \mathrm{eV} \cdot{ }^{16,17}$ However, the difference in WF between SWCNTs and a substrate is not sufficient to explain quantitatively the $E_{F}$ shift shown in the present work. Organic/metal interfaces manifest an interfacial dipole layer due to charge transfer, image charge formation, chemical interaction, interfacial state, and other factors. ${ }^{18}$ Since the chemical potential of electrons is much higher in silver than in the SWCNTs, electrons flow from Ag to a SWCNT to achieve equilibrium at the interface (Fig. 3). As a result, interfacial dipole moments are generated and the $E_{W}$ alignment shifts. The vacuum level (VL) shift depends on
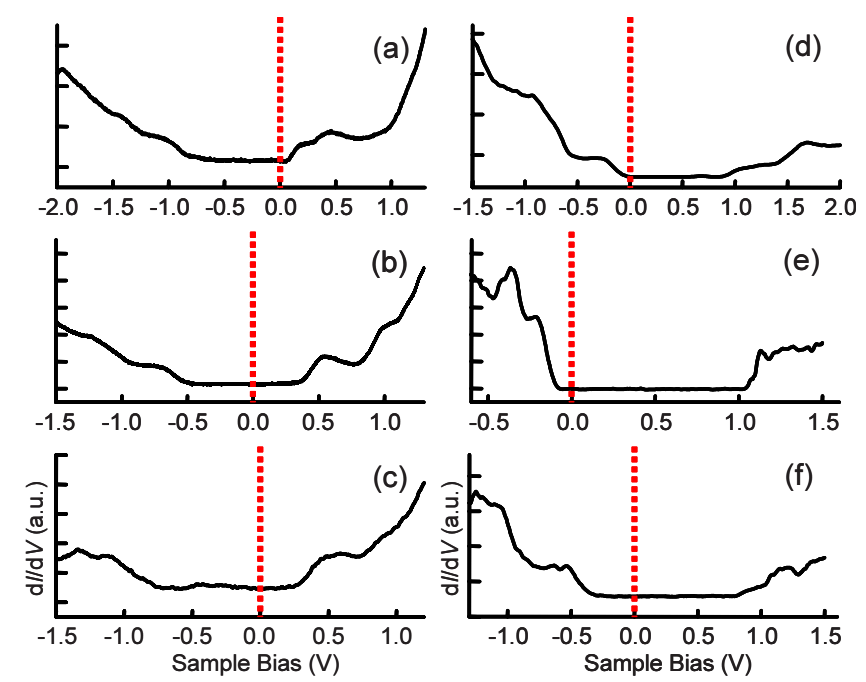

FIG. 2. (Color online) The $d I / d V$ spectra recorded on various SWCNTs: $[(\mathrm{a})-(\mathrm{c})]$ on $\mathrm{Ag}(100)$ and $[(\mathrm{d})-(\mathrm{f})]$ on $\mathrm{NaCl} / \mathrm{Ag}(100)$. The vertical dashed lines indicate the location of $E_{F}$.

the electron affinity of the SWCNTs, so those having different chiralities or different adsorption geometries can have a different degree of VL shift on $\mathrm{Ag}(100)$.

The WF of the surface changes when an insulating film is deposited on the metal surface. In the case of $\operatorname{Ag}(100)$, the WF is reduced to about $3.6-4.0 \mathrm{eV}$ by deposition of a $\mathrm{NaCl}$ film. ${ }^{19}$ If we consider only the WF values of the tube and the $\mathrm{NaCl}$ films, the $E_{F}$ of a SWCNT should be shifted much more to the CB. However, the direction of $E_{F}$ shift for the SWCNTs on $\mathrm{NaCl}$ in the experimental results was the opposite, i.e., to the VB. This means that the different WFs between SWCNT and substrate are not the dominant factors for determining the $E_{F}$ shift in this case.

What is the driving force of the $E_{F}$ shift in SWCNTs on an insulating film? The dipole moment at the interface seems likely to be responsible. When an insulating film interfaces with a metal surface, interface states, i.e., metal-induced gap states (MIGSs), develop at the interface. ${ }^{20,21}$ The presence of MIGS at the interface gives rise to a charge transfer across the interface, creating a dipole moment. The substrateinduced polarization of electrons in metal is also a reason for the interfacial dipole moment. In the case of $\mathrm{NaCl} / \mathrm{Ag}(100)$,

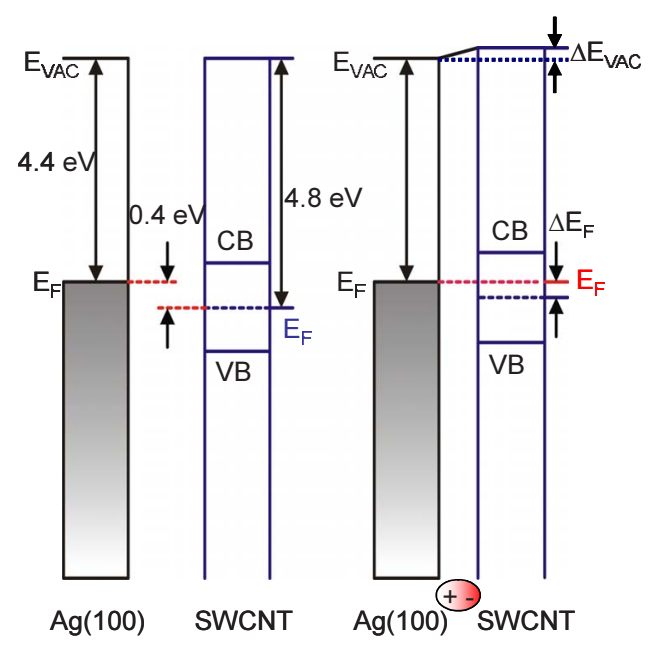

FIG. 3. (Color online) Schematic of interfacial energy of SWCNT/Ag(100) before (left) and after (right) contact. 


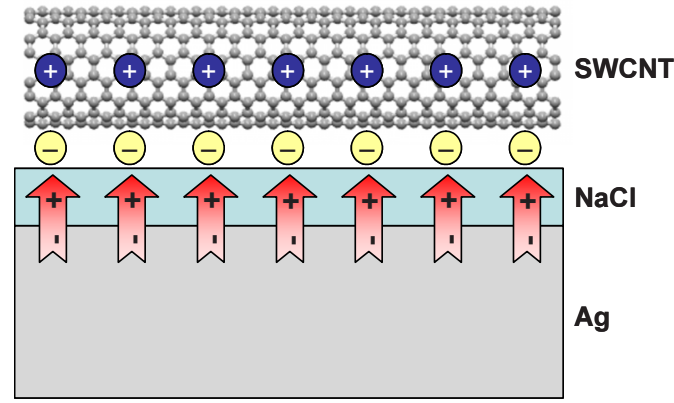

FIG. 4. (Color online) Schematic of a SWCNT on $\mathrm{NaCl} / \mathrm{Ag}(100)$ showing the dipole moments at the interface and their role in attracting electrons from the SWCNT.

the calculated results in Ref. 19 also revealed that an interfacial dipole moment developed at the interface due to an electron depletion in the insulating film in favor of the substrate. The direction of the electric field created in this way attracts electrons from the SWCNT to the substrate, which ultimately results in effective $p$-doping of the tube (Fig. 4). We believe that the effect of the dipole moment is not negligible on ultrathin insulating films and that this is the reason why the $E_{F}$ shift in the tube on the $\mathrm{NaCl}$ film is opposite to that on $\operatorname{Ag}(100)$ in spite of the lowered WF of the substrate. Since the electric field of interfacial dipole moment varies according to the thickness of the insulating film, ${ }^{21}$ we could expect that this might also affect the $E_{F}$ alignment of the SWCNTs. However, we could not compare the amount of the $E_{F}$ shift as a function of the thickness of the $\mathrm{NaCl}$ layers from our data. Most of our STS data were measured from the SWCNTs on the $\mathrm{NaCl}$ films of 1 or $2 \mathrm{ML}$ in thickness; for example, the STS data in Figs. 2(d)-2(f) were measured from the SWCNTs on the different $\mathrm{NaCl}$ films of the same thickness (2 ML). Depending on the SWCNTs, the $E_{F}$ of the tubes even on the $\mathrm{NaCl}$ films of the same thickness are distributed in a wide range as those on the silver substrate. So it is difficult to directly correlate the $E_{F}$ shift in various SWCNTs with the thickness of the $\mathrm{NaCl}$ layers from the current STS data.

To summarize, we examined the $E_{F}$ alignment of SWCNTs on $\mathrm{Ag}(100)$ and ultrathin $\mathrm{NaCl}$ films by means of LT-STM and STS. On pristine metal surface, the $E_{F}$ shift was influenced by the difference in the WFs of substrate and the nanotube, whereas the electric field created at the interface between the insulating $\mathrm{NaCl}$ film and the metal substrate was decisive in determining the $E_{F}$ of the SWCNT on the $\mathrm{NaCl}$ film. It is well known that the doping of SWCNTs is not easily achieved by conventional semiconductor processes such as ion implantation or chemical modification. Our results suggest one way of controlling the $E_{F}$ of SWCNTs and also reveal the importance of the nanometer-scale insulating layer in determining the $E_{F}$ alignment not only for SWCNTs but also for other kinds of molecular systems on insulating film.

The present work was supported by the Grant-in-Aid for Young Scientists (A), "Single-molecule chemistry on the single-walled carbon nanotubes," and partially by the Grantin-Aid for Scientific Research on Priority Areas, "Electron transport through a linked molecule in nano-scale," from the Ministry of Education, Culture, Sports, Science and Technology, and also in part by Global COE Program (Chemistry Innovation through Cooperation of Science and Engineering), MEXT, Japan.

${ }^{1}$ M. S. Dresselhaus, G. Dresselhaus, and P. Avouris, Carbon Nanotubes: Synthesis, Structure, Properties and Applications (Springer, Berlin, 2001).

${ }^{2}$ P. Avouris and J. Chen, Mater. Today 9, 46 (2006).

${ }^{3}$ J. W. G. Wilder, L. C. Venema, A. G. Rinzler, R. E. Smalley, and C. Dekker, Nature (London) 391, 59 (1998).

${ }^{4}$ S. G. Lemay, J. W. Janssen, M. van den Hout, M. Mooji, M. J. Bronikowski, P. A. Willis, R. E. Smalley, L. P. Kouwenhoven, and C. Dekker, Nature (London) 412, 617 (2001).

${ }^{5}$ M. Ouyang, J.-L. Huang, and C. M. Lieber, Annu. Rev. Phys. Chem. 53, 201 (2002).

${ }^{6}$ P. M. Albrecht and J. W. Lyding, Appl. Phys. Lett. 83, 5029 (2003).

${ }^{7}$ L. B. Ruppalt and J. W. Lyding, Nanotechnology 18, 215202 (2007).

${ }^{8}$ J. Repp, G. Meyer, S. Paavilainen, F. E. Olsson, and M. Persson, Science 312, 1196 (2006).

${ }^{9}$ J. Repp and G. Meyer, Appl. Phys. A: Mater. Sci. Process. 85, 399 (2006).

${ }^{10}$ J. Repp, G. Meyer, F. E. Olsson, and M. Persson, Science 305, 493 (2004).

${ }^{11}$ S. Clair, C. Rabot, Y. Kim, and M. Kawai, J. Vac. Sci. Technol. B 25, 1143 (2007).

${ }^{12}$ Y. Xue and S. Datta, Phys. Rev. Lett. 83, 4844 (1999).

${ }^{13}$ S. Okada and A. Oshiyama, Phys. Rev. Lett. 95, 206804 (2005).

${ }^{14}$ K. Hayashi, R. Arafune, S. Ueda, and S. Ushioda, Appl. Surf. Sci. 237, 296 (2004).

${ }^{15}$ H. B. Michaelson, J. Appl. Phys. 48, 4729 (1977)

${ }^{16}$ J. Zhao, J. Han, and J. P. Lu, Phys. Rev. B 65, 193401 (2002).

${ }^{17}$ V. Barone, J. E. Peralta, J. Uddin, and G. E. Scuseria, J. Chem. Phys. 124, 024709 (2006).

${ }^{18}$ H. Ishii, K. Sugiyama, E. Ito, and K. Seki, Adv. Mater. (Weinheim, Ger.) 11, 605 (1999).

${ }^{19}$ M. Pivetta, F. Patthey, M. Stengel, A. Baldereschi, and W.-D. Schneider, Phys. Rev. B 72, 115404 (2005).

${ }^{20}$ J. Goniakowski and C. Noguera, Interface Sci. 12, 93 (2004)

${ }^{21}$ L. Giordano, F. Cinquini, and G. Pacchioni, Phys. Rev. B 73, 045414 (2006) 